PROCEEDINGS OF THE

AMERICAN MATHEMATICAL SOCIETY

Volume 139, Number 3, March 2011, Pages 855-863

S 0002-9939(2010)10542-7

Article electronically published on August 18, 2010

\title{
LIMITS OF COALGEBRAS, BIALGEBRAS AND HOPF ALGEBRAS
}

\author{
A. L. AGORE \\ (Communicated by Martin Lorenz) \\ Dedicated to the memory of Professor S. Ianus
}

\begin{abstract}
We give the explicit construction of the product of an arbitrary family of coalgebras, bialgebras and Hopf algebras: it turns out that the product of an arbitrary family of coalgebras (resp. bialgebras, Hopf algebras) is the sum of a family of coalgebras (resp. bialgebras, Hopf algebras). The equalizers of two morphisms of coalgebras (resp. bialgebras, Hopf algebras) are also described explicitly. As a consequence the categories of coalgebras, bialgebras and Hopf algebras are shown to be complete, and a complete description for limits in the above categories is given.
\end{abstract}

\section{INTRODUCTION}

It is well known that the category $k$-Alg of $k$-algebras is complete and cocomplete; that is, any functor $F: I \rightarrow k$-Alg has a limit and a colimit for all small categories $I$. This is immediately implied by the existence of products, coproducts, equalizers and coequalizers in the category $k$-Alg. The categories of coalgebras, bialgebras or Hopf algebras have arbitrary coproducts and coequalizers (see [5, Proposition 1.4.19], 4, Proposition 2.10], 8, Corollary 2.6.6] and [2, Remark 2.1, Theorem 2.2]); hence these categories are cocomplete. Related to the question of whether these categories are complete (i.e. if they have arbitrary products and equalizers), we could not find similar results in the classical Hopf algebra textbooks ([1, 9]), not even in the more recent ones (4, 5]). For example, [5, Propositon 1.4.21] proves only the existence of finite products (namely the tensor product of coalgebras) and only in the category of cocommutative coalgebras, as a dual result to the one concerning commutative algebras.

In this paper we shall fill this gap: using the fact that the forgetful functor from the category of coalgebras to the category of vector spaces has a right adjoint, namely the so-called cofree coalgebra, we shall construct explicitly the product of an arbitrary family of coalgebras. As a consequence, the product of an arbitrary family of bialgebras and Hopf algebras is constructed. The equalizers of two morphisms of

Received by the editors September 16, 2009 and, in revised form, April 14, 2010.

2000 Mathematics Subject Classification. Primary 16W30, 18A30, 18A40.

Key words and phrases. Product of coalgebras, bialgebras, Hopf algebras.

The author acknowledges partial support from CNCSIS grant 24/28.09.07 of PN II "Groups, quantum groups, corings and representation theory".

(C)2010 American Mathematical Society Reverts to public domain 28 years from publication 
coalgebras (bialgebras, Hopf algebras) are also described explicitly. Thus we shall obtain that the categories of coalgebras, bialgebras and Hopf algebras are complete.

Throughout this paper, $k$ will be a field. Unless specified otherwise, all vector spaces, algebras, coalgebras, bialgebras, tensor products and homomorphisms are over $k$. Our notation for the standard categories is as follows: ${ }_{k} \mathcal{M}$ ( $k$-vector spaces), $k$-Alg (associative unital $k$-algebras), $k$-CoAlg (coalgebras over $k$ ), $k$-BiAlg (bialgebras over $k$ ), $k$-HopfAlg (Hopf algebras over $k$ ), $\mathcal{M}^{C}$ (right $C$-comodules). For a coalgebra $C$, we will use Sweedler's $\Sigma$-notation, that is, $\Delta(c)=c_{(1)} \otimes c_{(2)}$, $(I \otimes \Delta) \Delta(c)=c_{(1)} \otimes c_{(2)} \otimes c_{(3)}$, etc. Given a vector space $V,(K(V), p)$ stands for the cofree coalgebra on $V$, where $K(V)$ is a coalgebra and $p: K(V) \rightarrow V$ is a $k$-linear map. We refer to [1, [5, 9] for further details concerning Hopf algebras. A category $\mathcal{C}$ is called (co) complete if any functor $F: I \rightarrow \mathcal{C}$ has (co)limits, where $I$ is a small category. A category $\mathcal{C}$ is (co)complete if and only if $\mathcal{C}$ has (co)equalizers of all pairs of arrows and all (co)products [7, Theorem 6.10]. Given a morphism $f \in \mathcal{C}$ we denote by $\operatorname{dom}(f)$ and $\operatorname{cod}(f)$ the domain, respectively the codomain, of $f$. If $\mathcal{C}$ is a small category we denote by $\operatorname{Hom}(\mathcal{C})$ the set of all morphisms of $\mathcal{C}$.

\section{Limits For COALGEBRAs, Bialgebras AND Hopf ALgebras}

First, we explicitly construct the product of an arbitrary family of coalgebras.

Theorem 1.1. The category $k$-CoAlg of coalgebras has arbitrary products and equalizers. In particular, the category $k$-CoAlg of coalgebras is complete.

Proof. Let $f, g: C \rightarrow D$ be two coalgebra maps and $S:=\{c \in C \mid f(c)=g(c)\}$, which is a $k$-subspace of $C$. Let $E$ be the sum of all subcoalgebras of $C$ included in $S$. Note that the family of subcoalgebras of $C$ included in $S$ is not empty since it contains the null coalgebra. It is immediate that $E$ is a subcoalgebra of $C$. We shall prove that $(E, i)$ is the equalizer of the pair $(f, g)$ in $k$-CoAlg, where $i: E \rightarrow C$ is the canonical inclusion. Let $E^{\prime}$ be a coalgebra and $h: E^{\prime} \rightarrow C$ a coalgebra map such that $f \circ h=g \circ h$. Then $f(h(x))=g(h(x))$ for all $x \in E^{\prime}$; hence $h\left(E^{\prime}\right) \in S$. Since $h\left(E^{\prime}\right)$ is a subcoalgebra in $C$ we obtain $h\left(E^{\prime}\right) \subseteq E$. Thus there exists a unique coalgebra map $h: E^{\prime} \rightarrow E$ such that $i \circ h=h$. Hence $(E, i)$ is the equalizer of the pair $(f, g)$ in the category $k$-CoAlg of coalgebras.

Now let $\left(C_{i}\right)_{i \in I}$ be a family of coalgebras and $\left(\prod_{i \in I} C_{i},\left(\pi_{i}\right)_{i \in I}\right)$ be the product of the $k$-modules $\left(C_{i}\right)_{i \in I}$. Let $\left(K\left(\prod_{i \in I} C_{i}\right), p\right)$ be the cofree coalgebra over the vector space $\prod_{i \in I} C_{i}$.

Let $D$ be the sum of all subcoalgebras $E$ of $K\left(\prod_{i \in I} C_{i}\right)$ such that $\pi_{i} \circ p \circ j_{E}$ is a coalgebra map for all $i \in I$, where $j_{E}: E \rightarrow K\left(\prod_{i \in I} C_{i}\right)$ is the canonical inclusion. The family of subcoalgebras of $E$ satisfying this property is nonempty since it contains the null coalgebra. The $k$-linear map $\pi_{i} \circ p \circ j: D \rightarrow C_{i}$ is a coalgebra map for all $i \in I$, where $j: D \rightarrow K\left(\prod_{i \in I} C_{i}\right)$ is the canonical inclusion. We shall prove that $\left(D,\left(\pi_{i} \circ p \circ j\right)_{i \in I}\right)$ is the product of the family of coalgebras $\left(C_{i}\right)_{i \in I}$ in $k$-CoAlg.

Indeed, let $D^{\prime}$ be a coalgebra and $g_{i}: D^{\prime} \rightarrow C_{i}, i \in I$, be a family of coalgebra maps. Using the universal property of the product in ${ }_{k} \mathcal{M}$ we obtain that there exists a unique $k$-linear map $\bar{g}: D^{\prime} \rightarrow \prod_{i \in I} C_{i}$ such that $\pi_{i} \circ \bar{g}=g_{i}$, for all $i \in I$. Furthermore, since $\left(K\left(\prod_{i \in I} C_{i}\right), p\right)$ is the cofree coalgebra over the $k$ module $\prod_{i \in I} C_{i}$, there exists a unique coalgebra map $\bar{f}: D^{\prime} \rightarrow K\left(\prod_{i \in I} C_{i}\right)$ such 
that $p \circ \bar{f}=\bar{g}$. Thus we have the following commutative diagram:

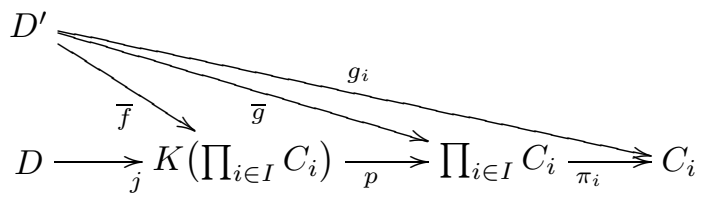

It follows that $\left(\pi_{i} \circ p\right)(\bar{f}(x))=g_{i}(x)$, for all $x \in D^{\prime}$ and $i \in I$. So, since each $g_{i}$ is a coalgebra map, we have $\bar{f}\left(D^{\prime}\right) \subseteq D$. Hence, we proved that for any coalgebra $D^{\prime}$ and any family $g_{i}: D^{\prime} \rightarrow C_{i}, i \in I$, of coalgebra maps there exists a coalgebra map $\bar{f}: D^{\prime} \rightarrow D$ such that $\left(\pi_{i} \circ p \circ j\right) \circ \bar{f}=g_{i}$, for all $i \in I$. Let $h: D^{\prime} \rightarrow D$ be another coalgebra map such that $\left(\pi_{i} \circ p \circ j\right) \circ h=g_{i}$ for all $i \in I$. From the uniqueness of $\bar{g}$ we obtain $p \circ j \circ h=\bar{g}$. Moreover, from the uniqueness of $\bar{f}$ we obtain $j \circ h=\bar{f}$, hence $h=\bar{f}$. Thus $\left(D,\left(\pi_{i} \circ p \circ j\right)_{i \in I}\right)$ is the product of the family $\left(C_{i}\right)_{i \in I}$ in the category $k$-CoAlg of coalgebras.

Remark 1.2. In 3, Lemma 1.1.3] a description for the equalizers in the category $k$-HopfAlg is given. We can use the same method in order to obtain another description for the equalizer of a pair of coalgebra (or bialgebra) maps. Let $f, g: C \rightarrow D$ be two coalgebra maps. It can be easily proved that $(E, i)$ is the equalizer of the pair $(f, g)$ in the category $k$-CoAlg of coalgebras, where

$$
E=\left\{c \in C \mid c_{(1)} \otimes f\left(c_{(2)}\right) \otimes c_{(3)}=c_{(1)} \otimes g\left(c_{(2)}\right) \otimes c_{(3)}\right\}
$$

and $i: E \rightarrow C$ is the canonical inclusion. This equivalent description of equalizers in the category $k$-CoAlg will turn out to be more efficient for computations.

Example 1.3. Let $G$ be a multiplicative group and $k G$ be the $k$-vector space with basis $\{g \mid g \in G\}$ endowed with the classical coalgebra structure : $\Delta(g)=g \otimes g$ and $\varepsilon(g)=1$ for all $g \in G$. Thus any element $x \in k G$ has the form $x=\sum_{g \in G} k_{g} g$, where $\left(k_{g}\right)_{g \in G}$ is a family of elements in $k$ with only a finite number of nonzero elements. We use the formal notation $x^{-1}:=\sum_{g \in G} k_{g} g^{-1}$ and $0^{-1}=0$. Consider the coalgebra maps $f=I d_{k G}$ and $h: k G \rightarrow k G$ given by $h(g)=g^{-1}$ for all $g \in G$. Then, in light of the above remark, it follows that the equalizer of the morphisms $(f, g)$ is given by the pair $(E, i)$, where $E=\left\{x \in k G \mid x \otimes x \otimes x=x \otimes x^{-1} \otimes x\right\}$ and $i$ is the canonical inclusion.

As an easy consequence of [6, Chapter $5 \S 2$, Theorem 1] we obtain the following description for limits in the category $k$-CoAlg of coalgebras.

Remark 1.4. Let $J$ be a small category, $F: J \rightarrow k$-CoAlg be a functor, $\left(\Pi_{j \in J} F(j)\right.$, $\left.\left(p_{j}\right)_{j \in J}\right),\left(\Pi_{u \in H o m(J)} F(\operatorname{cod}(u)),\left(p_{u}\right)_{u \in H o m(J)}\right)$ be the product in $k$-CoAlg of the families $(F(j))_{j \in J}$, respectively $(F(\operatorname{cod}(u)))_{u \in H o m(J)}$, and $f, g: \Pi_{j \in J} F(j)$ $\rightarrow \Pi_{u \in \operatorname{Hom}(J)} F(\operatorname{cod}(u))$ be the unique coalgebra maps such that $p_{u} \circ f=p_{\operatorname{cod}(u)}$ and $p_{u} \circ g=F(u) \circ p_{\operatorname{dom}(u)}$ for all $u \in \operatorname{Hom}(J)$. We define

$$
D=\left\{x \in \Pi_{j \in J} F(j) \mid x_{(1)} \otimes f\left(x_{(2)}\right) \otimes x_{(3)}=x_{(1)} \otimes g\left(x_{(2)}\right) \otimes x_{(3)}\right\} .
$$

Then the pair $\left(D,\left(\varphi_{j}=p_{j} \circ e\right)_{j \in J}\right)$ is the limit of the functor $F$, where $e: D \rightarrow$ $\Pi_{j \in J} F(j)$ is the canonical inclusion.

In what follows we will make use of Theorem 1.1 in order to construct the product in the category of $k$-BiAlg of bialgebras. 
Theorem 1.5. The category $k$-BiAlg of bialgebras has arbitrary products and equalizers. In particular, the category $k$-BiAlg of bialgebras is complete.

Proof. Let $\left(B_{i}, m_{i}, \eta_{i}, \Delta_{i}, \varepsilon_{i}\right)_{i \in I}$ be a family of bialgebras and let $\left(\left(\prod_{i \in I} B_{i}, \Delta, \varepsilon\right)\right.$, $\left.\left(\pi_{i}\right)_{i \in I}\right)$ be the product of this family in the category $k$-CoAlg of coalgebras. Since $\left(B_{i}, m_{i}, \eta_{i}, \Delta_{i}, \varepsilon_{i}\right)$ is a bialgebra it follows that $m_{i}: B_{i} \otimes B_{i} \rightarrow B_{i}$ and $\eta_{i}: k \rightarrow B_{i}$ are coalgebra maps for all $i \in I$. Then there exists a unique coalgebra map $\eta: k \rightarrow$ $\prod_{i \in I} B_{i}$ such that the diagram

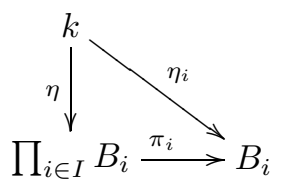

is commutative for all $i \in I$. Also there exists a unique coalgebra map $m: \prod_{i \in I} B_{i} \otimes$ $\prod_{i \in I} B_{i} \rightarrow \prod_{i \in I} B_{i}$ for which the diagram

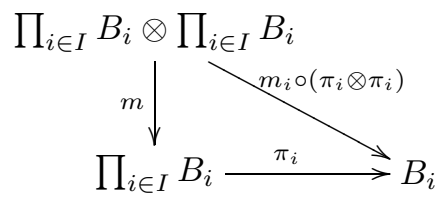

is commutative for all $i \in I$.

First, we will prove that $\left(\prod_{i \in I} B_{i}, m, \eta\right)$ is a $k$-algebra. Since $\pi_{i} \circ m \circ(m \otimes I d)$ is a coalgebra map, by the universal property of the product we obtain that there exists a unique coalgebra map $\psi:\left(\prod_{i \in I} B_{i}\right)^{\otimes 3} \rightarrow \prod_{i \in I} B_{i}$ such that the diagram

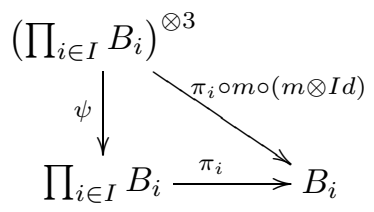

is commutative for all $i \in I$. It is easy to see that the coalgebra map $m \circ(m \otimes I d)$ makes the above diagram commute. Thus, using the uniqueness of $\psi$, in order to prove that $m \circ(m \otimes I d)=m \circ(I d \otimes m)$ it is enough to show that $\pi_{i} \circ m \circ(m \otimes I d)=$ $\pi_{i} \circ m \circ(I d \otimes m)$ for all $i \in I$. We have

$$
\begin{aligned}
& \pi_{i} \circ m \circ(m \otimes I d) \stackrel{\text { 五 }}{=} m_{i} \circ\left(\pi_{i} \otimes \pi_{i}\right) \circ(m \otimes I d) \\
& =m_{i} \circ\left(\left(\pi_{i} \circ m\right) \otimes \pi_{i}\right) \\
& \underline{\underline{\underline{2}}} \quad m_{i} \circ\left[\left(m_{i} \circ\left(\pi_{i} \otimes \pi_{i}\right)\right) \otimes \pi_{i}\right] \\
& =m_{i} \circ\left(m_{i} \otimes I d\right) \circ\left(\pi_{i} \otimes \pi_{i} \otimes \pi_{i}\right) \\
& =m_{i} \circ\left(I d \otimes m_{i}\right) \circ\left(\pi_{i} \otimes \pi_{i} \otimes \pi_{i}\right) \\
& =m_{i} \circ\left[\pi_{i} \otimes\left(m_{i} \circ\left(\pi_{i} \otimes \pi_{i}\right)\right)\right] \\
& \text { 胥 } \quad m_{i} \circ\left(\pi_{i} \otimes\left(\pi_{i} \circ m\right)\right) \\
& =m_{i} \circ\left(\pi_{i} \otimes \pi_{i}\right) \circ(I d \otimes m) \\
& \stackrel{\underline{\underline{2}}}{=} \pi_{i} \circ m \circ(I d \otimes m) \text {. }
\end{aligned}
$$

Hence $m \circ(m \otimes I d)=m \circ(I d \otimes m)$; i.e. $m$ is associative. 
Now consider the coalgebra map $\pi_{i} \circ m \circ(\eta \otimes I d)$. From the universal property of the product, we obtain that there exists a unique coalgebra map $\varphi: k \otimes \prod_{i \in I} B_{i} \rightarrow$ $B_{i}$ such that the diagram

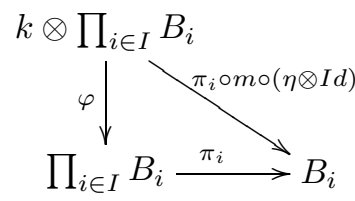

is commutative for all $i \in I$. By the argument above, in order to prove that $m \circ(\eta \otimes I d)=s$ it will be enough to show that $\pi_{i} \circ m \circ(\eta \otimes I d)=\pi_{i} \circ s$, where we denote by $s$ the scalar multiplication. We have

$$
\begin{aligned}
\pi_{i} \circ m \circ(\eta \otimes I d) & \stackrel{\underline{\underline{\underline{2}}}}{=} m_{i} \circ\left(\pi_{i} \otimes \pi_{i}\right) \circ(\eta \otimes I d) \\
= & m_{i} \circ\left(\left(\pi_{i} \circ \eta\right) \otimes \pi_{i}\right) \\
\underline{\underline{\underline{\underline{D}}}} & m_{i} \circ\left(\eta_{i} \otimes \pi_{i}\right) \\
= & m_{i} \circ\left(\eta_{i} \otimes I d\right) \circ\left(I d \otimes \pi_{i}\right) \\
= & s \circ\left(I d \otimes \pi_{i}\right) .
\end{aligned}
$$

Furthermore, let $k_{1} \otimes b \in k \otimes \prod_{i \in I} B_{i}$. Having in mind that $\pi_{i}$ is a $k$-linear map we obtain

$$
\begin{aligned}
s \circ\left(I d \otimes \pi_{i}\right)\left(k_{1} \otimes b\right) & =k_{1} \pi_{i}(b) \\
& =\pi_{i}\left(k_{1} b\right) \\
& =\pi_{i} \circ s\left(k_{1} \otimes b\right) .
\end{aligned}
$$

Thus we have proved that $\pi_{i} \circ m \circ(\eta \otimes I d)=\pi_{i} \circ s$. In the same way it follows that $m \circ(I d \otimes \eta)=s$. Hence $\left(\prod_{i \in I} B_{i}, m, \eta\right)$ is an algebra, and since $m$ and $\eta$ are coalgebra maps, we obtain that $\left(\prod_{i \in I} B_{i}, m, \eta, \Delta, \varepsilon\right)$ is actually a bialgebra.

To end the proof we still need to show that $\left(\prod_{i \in I} B_{i}, m, \eta, \Delta, \varepsilon\right)$ is the product of the family $\left(B_{i}, m_{i}, \eta_{i}, \Delta_{i}, \varepsilon_{i}\right)_{i \in I}$ in the category $k$-BiAlg. Let $\left(B, m_{B}, \eta_{B}, \Delta_{B}, \varepsilon_{B}\right)$ be a bialgebra and $\left(g_{i}\right)_{i \in I}$ be a family of bialgebra maps, $g_{i}: B \rightarrow B_{i}$ for all $i \in I$. From the universal property of the product, we obtain that there exists a unique coalgebra map $\theta: B \rightarrow \prod_{i \in I} B_{i}$ such that the following diagram commutes:

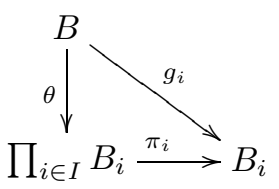

We only need to prove that $\theta$ is also an algebra map. By the argument used above, it is enough to show that

$$
\pi_{i} \circ \theta \circ m_{B}=\pi_{i} \circ m \circ(\theta \otimes \theta) \quad \text { and } \quad \pi_{i} \circ \theta \circ \eta_{B}=\pi_{i} \circ \eta
$$


Having in mind that $g_{i}$ is an algebra map, we have

$$
\begin{aligned}
& \pi_{i} \circ m \circ(\theta \otimes \theta) \stackrel{\text { ? }}{=} m_{i} \circ\left(\pi_{i} \otimes \pi_{i}\right) \circ(\theta \otimes \theta) \\
& =m_{i} \circ\left(\left(\pi_{i} \circ \theta\right) \otimes\left(\pi_{i} \circ \theta\right)\right) \\
& \text { 皇 } m_{i} \circ\left(g_{i} \otimes g_{i}\right) \\
& =g_{i} \circ m_{B} \\
& \text { 惡 } \pi_{i} \circ \theta \circ m_{B} \text {. }
\end{aligned}
$$

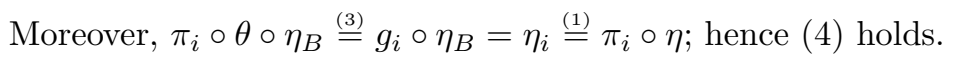

In what follows we construct equalizers. Let $\left(A, m_{A}, \eta_{A}, \Delta_{A}, \varepsilon_{A}\right)$ and $\left(B, m_{B}\right.$, $\left.\eta_{B}, \Delta_{B}, \varepsilon_{B}\right)$ be two bialgebras and $f, g: B \rightarrow A$ be two bialgebra maps. We denote this by $S:=\{b \in B \mid f(b)=g(b)\}$. Let $D$ be the sum of all subcoalgebras of $B$ contained in $S$. We already noticed before that the family of subcoalgebras of $B$ with this property is nonempty and that $D$ is a subcoalgebra of $B$. The pair $(D, i)$ is the equalizer of the morphisms $(f, g)$ in $k$-BiAlg, where $i: D \rightarrow B$ is the canonical inclusion. We only need to prove that $D$ is actually a subbialgebra of $B$. Consider $q=\sum_{k=1}^{n} d_{i_{k}} \otimes d_{j_{k}} \in D \otimes D$. We then have

$$
\begin{aligned}
m_{A} \circ(f \otimes f)(q) & =m_{A}\left(\sum_{k=1}^{n} f\left(d_{i_{k}}\right) \otimes f\left(d_{j_{k}}\right)\right) \\
& =m_{A}\left(\sum_{k=1}^{n} g\left(d_{i_{k}}\right) \otimes g\left(d_{j_{k}}\right)\right) \\
& =m_{A} \circ(g \otimes g)(q) .
\end{aligned}
$$

Now having in mind that $f$ and $g$ are algebra maps we obtain $f\left(m_{B}(D \otimes D)\right)=$ $g\left(m_{B}(D \otimes D)\right)$. Hence $m_{B}(D \otimes D) \subseteq S$, and since $m_{B}(D \otimes D)$ is a subcoalgebra, it follows that $m_{B}(D \otimes D) \subseteq D$. Thus $D$ is a subbialgebra of $B$, and it can be shown as in Theorem 1.1 that the pair $(D, i)$ is the equalizer of the morphisms $(f, g)$ in $k$-BiAlg.

As remarked before, we can obtain a description for the equalizers in $k$-BiAlg similar to the one in Remark 1.2. Thus, we have the following description for limits in $k$-BiAlg:

Remark 1.6. Let $J$ be a small category, $F: J \rightarrow k$-BiAlg be a functor, $\left(\Pi_{j \in J} F(j)\right.$, $\left.\left(p_{j}\right)_{j \in J}\right),\left(\Pi_{u \in \operatorname{Hom}(J)} F(\operatorname{cod}(u)),\left(p_{u}\right)_{u \in H o m(J)}\right)$ be the product in $k$-BiAlg of the families $(F(j))_{j \in J}$, respectively $(F(\operatorname{cod}(u)))_{u \in H o m(J)}$, and $f, g: \Pi_{j \in J} F(j) \rightarrow$ $\Pi_{u \in \operatorname{Hom}(J)} F(\operatorname{cod}(u))$ be the unique bialgebra maps such that $p_{u} \circ f=p_{\operatorname{cod}(u)}$ and $p_{u} \circ g=F(u) \circ p_{\operatorname{dom}(u)}$ for all $u \in \operatorname{Hom}(J)$. We define

$$
D=\left\{x \in \Pi_{j \in J} F(j) \mid x_{(1)} \otimes f\left(x_{(2)}\right) \otimes x_{(3)}=x_{(1)} \otimes g\left(x_{(2)}\right) \otimes x_{(3)}\right\} .
$$

Then the pair $\left(D,\left(\varphi_{j}=p_{j} \circ e\right)_{j \in J}\right)$ is the limit of the functor $F$, where $e: D \rightarrow$ $\Pi_{j \in J} F(j)$ is the canonical inclusion.

Theorem 1.7. The category $k$-HopfAlg of Hopf algebras has arbitrary products and equalizers. In particular, the category $k$-HopfAlg of Hopf algebras is complete.

Proof. Let $\left(H_{i}, m_{i}, \eta_{i}, \Delta_{i}, \varepsilon_{i}, S_{i}\right)_{i \in I}$ be a family of Hopf algebras and let ( $B:=$ $\left.\left.\prod_{i \in I} H_{i}, \Delta, \varepsilon, m, \eta\right),\left(\pi_{i}\right)_{i \in I}\right)$ be the product of this family in the category $k$-BiAlg 
of bialgebras. The universal property of the product yields a unique bialgebra map $S: B^{o p, c o p} \rightarrow B$ such that the following diagram commutes for all $i \in I$ :

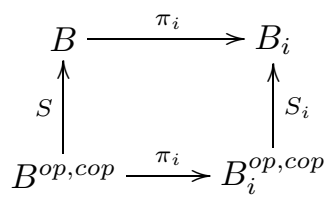

Let $H$ be the sum of all subcoalgebras $C$ of the bialgebra $B$ such that $S\left(c_{(1)}\right) c_{(2)}=$ $c_{(1)} S\left(c_{(2)}\right)=\eta \circ \varepsilon(c)$ for all $c \in C$. The family of subcoalgebras $C$ satisfying the above property is nonempty by the same argument used in the proof of Theorem 1.1. Moreover, it is easy to see that

$$
S\left(h_{(1)}\right) h_{(2)}=h_{(1)} S\left(h_{(2)}\right)=\eta \circ \varepsilon(h)
$$

for all $h \in H$. We will prove that $H$ is a bialgebra, and it will follow by (6) that $H$ is actually a Hopf algebra with the antipode $S_{\mid H}$. First note that $\eta(k)=k 1_{B} \subseteq H$. Let $h, g \in H$. We then have

$$
\begin{aligned}
S\left((h g)_{(1)}\right)(h g)_{(2)} & =S\left(h_{(1)} g_{(1)}\right) h_{(2)} g_{(2)} \\
& =S\left(g_{(1)}\right) S\left(h_{(1)}\right) h_{(2)} g_{(2)} \\
& =S\left(g_{(1)}\right)(\eta \circ \varepsilon)(h) g_{(2)} \\
& =(\eta \circ \varepsilon(h))(\eta \circ \varepsilon(g))=\eta \circ \varepsilon(h g) .
\end{aligned}
$$

In the same way it can be proved that $(h g)_{(1)} S\left((h g)_{(2)}\right)=\eta \circ \varepsilon(h g)$. Thus $h g \in H$, and $H$ is indeed a bialgebra. In order to conclude that $S_{\mid H}$ is an antipode for $H$ we need to prove that $S(H) \subseteq H$. Let $h \in H$; we obtain

$$
\begin{aligned}
S\left(S(h)_{(1)}\right) S(h)_{(2)} & =S\left(S\left(h_{(2)}\right)\right) S\left(h_{(1)}\right) \\
& =S\left(h_{(1)} S\left(h_{(2)}\right)\right) \\
& =S(\eta \circ \varepsilon(h)) \\
& =\eta \circ \varepsilon(h)=\eta \circ \varepsilon(S(h)) .
\end{aligned}
$$

A similar computation shows that we also have $S(h)_{(1)} S\left(S(h)_{(2)}\right)=\eta \circ \varepsilon(S(h))$ for all $h \in H$. Hence $H$ is a Hopf algebra with $S_{\mid H}$ as antipode.

To end the proof we still need to show that $\left(\left(H, m, \eta, \Delta, \varepsilon, S_{\mid H}\right),\left(q_{i}\right)_{i \in I}\right)$ is the product of the family $\left(H_{i}, m_{i}, \eta_{i}, \Delta_{i}, \varepsilon_{i}, S_{i}\right)_{i \in I}$ in the category $k$-HopfAlg, where $q_{i}:=\pi_{i} \circ j$ for all $i \in I$ and $j: H \rightarrow B$ is the canonical inclusion.

Let $K$ be a Hopf algebra with antipode $S_{K}$ and $f_{i}: K \rightarrow H_{i}$ be a family of Hopf algebra maps for all $i \in I$. Since $B$ is the product in $k$-BiAlg of the above family of Hopf algebras, there exist a unique morphism of bialgebras $\bar{f}: K \rightarrow B$ such that the following diagram commutes:

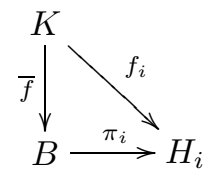


Using the fact that $f_{i}$ is a Hopf algebra map we have

$$
\begin{aligned}
& \pi_{i} \circ S \circ \bar{f} \stackrel{\underline{\underline{5}}}{=} S_{i} \circ \pi_{i} \circ \bar{f} \\
& \text { 金 } S_{i} \circ f_{i} \\
& =f_{i} \circ S_{K} \\
& \text { 国 } \pi_{i} \circ \bar{f} \circ S_{K}
\end{aligned}
$$

for all $i \in I$. By the same argument used in the proof of Theorem 1.5 it follows that

$$
S \circ \bar{f}=\bar{f} \circ S_{K}
$$

Thus, for all $k \in K$ we have

$$
\begin{aligned}
S\left(\bar{f}(k)_{(1)}\right) \bar{f}(k)_{(2)} & =S\left(\bar{f}\left(k_{(1)}\right)\right) \bar{f}\left(k_{(2)}\right) \\
& \stackrel{\underline{\underline{\text { g }}}}{f}\left(S_{K}\left(k_{(1)}\right)\right) \bar{f}\left(k_{(2)}\right) \\
& =\bar{f}\left(S_{K}\left(k_{(1)}\right) k_{(2)}\right) \\
& =\bar{f}(k) .
\end{aligned}
$$

Hence $\bar{f}(K) \subseteq H$. Thus, we obtained a unique Hopf algebra map $\bar{f}: K \rightarrow H$ such that $q_{i} \circ \bar{f}=f_{i}$ for all $i \in I$.

Now, since the forgetful functor $U: k$-HopfAlg $\rightarrow k$-BiAlg has a left adjoint (see [10]), it follows that, in particular, $U$ preserves products. That is, $H=B$ and the map $S$ obtained in (5) is actually an antipod for $B$. Thus, $((B, m, \eta, \Delta, \varepsilon, S)$, $\left.\left(\pi_{i}\right)_{i \in I}\right)$ is the product of the family $\left(H_{i}, m_{i}, \eta_{i}, \Delta_{i}, \varepsilon_{i}, S_{i}\right)_{i \in I}$ in the category $k$ HopfAlg.

Now let $f, g: H \rightarrow K$ be two Hopf algebra morphisms and let $S:=\{h \in$ $H \mid f(h)=g(h)\}$, which is just a $k$-subspace of $H$. Let $D$ be the sum of all subcoalgebras of $H$ contained in $S$. Again, the family of subcoalgebras of $H$ included in $S$ is not empty by the same argument used in Theorem 1.1. A simple computation shows that $D$ is in fact a Hopf subalgebra of $H$. Moreover, $(D, i)$ is the equalizer in the category $k$-HopfAlg of the pair $(f, g)$, where $i: D \rightarrow H$ is the canonical inclusion.

Remark 1.8. Let $J$ be a small category, $F: J \rightarrow k$-HopfAlg be a functor, $\left(\Pi_{j \in J} F(j)\right.$, $\left.\left(p_{j}\right)_{j \in J}\right), \quad\left(\Pi_{u \in \operatorname{Hom}(J)} F(\operatorname{cod}(u)),\left(p_{u}\right)_{u \in H o m(J)}\right)$ be the product in $k$-HopfAlg of the families $(F(j))_{j \in J}$, respectively $(F(\operatorname{cod}(u)))_{u \in H o m(J)}$, and $f, g: \Pi_{j \in J} F(j) \rightarrow$ $\Pi_{u \in H o m(J)} F(\operatorname{cod}(u))$ be the unique Hopf algebra maps such that $p_{u} \circ f=p_{\operatorname{cod}(u)}$ and $p_{u} \circ g=F(u) \circ p_{\operatorname{dom}(u)}$ for all $u \in \operatorname{Hom}(J)$. We define

$$
D=\left\{x \in \Pi_{j \in J} F(j) \mid x_{(1)} \otimes f\left(x_{(2)}\right) \otimes x_{(3)}=x_{(1)} \otimes g\left(x_{(2)}\right) \otimes x_{(3)}\right\} .
$$

Then the pair $\left(D,\left(\varphi_{j}=p_{j} \circ e\right)_{j \in J}\right)$ is the limit of the functor $F$, where $e: D \rightarrow$ $\Pi_{j \in J} F(j)$ is the canonical inclusion.

The key role in the construction of the product in the category of coalgebras was played by the fact that the forgetful functor from the category of coalgebras to the category of vector spaces has a right adjoint. It is therefore natural to ask if the conclusion remains true for the category of $R$-corings (4]). Let $R$ be a ring, $R$-Corings be the category of $R$-corings and ${ }_{R} \mathcal{M}_{R}$ be the category of $R$-bimodules. 
Problem. Does there exist a right adjoint for the forgetful functor $F: R-$ Corings $\rightarrow{ }_{R} \mathcal{M}_{R}$ ?

\section{ACKNOWLEDGEMENT}

The author wishes to thank Professor Gigel Militaru, who suggested the problems studied here, for his great support and for the useful comments from which this manuscript has benefitted.

\section{REFERENCES}

[1] E. Abe, Hopf Algebras, Cambridge University Press, Cambridge, 1977. MR594432 (83a:16010)

[2] A.L. Agore, Categorical constuctions for Hopf algebras, to appear in Commun. in Algebra, arXiv:0905.2613v3

[3] N. Andruskiewitsch, J. Devoto, Extensions of Hopf algebras, Algebra i Analiz 7 (1995), no. 1, 22-61. MR:1334152 (96f:16044)

[4] T. Brzeziński, R. Wisbauer, Corings and comodules, Cambridge University Press, 2003. MR2012570 (2004k:16093)

[5] S. Dăscălescu, C. Năstăsescu, Ş. Raianu, Hopf algebras. An introduction, Pure and Applied Math., 235, Marcel Dekker, New York, 2001. MR.1786197 (2001j:16056)

[6] Mac Lane, S., Categories for the working mathematician, Graduate Texts in Math., Vol. 5, Springer, New York, 1971. MR0354798 (50:7275)

[7] B. Pareigis, Advanced Algebra. Available at: http://www.mathematik.uni-muenchen.de/ pareigis/Vorlesungen/01WS/advalg.pdf

[8] B. Pareigis, Lectures on quantum groups and noncommutative geometry. Available at: http://www.mathematik.uni-muenchen.de/ pareigis/Vorlesungen/02SS/QGandNCG.pdf

[9] M.E. Sweedler, Hopf Algebras, Benjamin, New York, 1969. MR0252485 (40:5705)

[10] M. Takeuchi, Free Hopf algebras generated by coalgebras, J. Math. Soc. Japan 23 (1971), 561-582 MR0292876(45:1958)

Faculty of Mathematics and Computer Science, University of Bucharest, Str. Academiei 14, RO-010014 Bucharest 1, Romania - and - Department of Mathematics, Academy of Economic Studies, Piata Romana 6, RO-010374 Bucharest 1, Romania

E-mail address: ana.agore@fmi.unibuc.ro 